\title{
La divulgación científica en prehistoria llevada a personas con discapacidad intelectual: una experiencia particular
}

\section{Scientific outreach about Prehistory with intellectually disabled people: a particular experience}

JUAN F. GIBAJA

Institució Milà i Fontanals (IMF-CSIC), Grupo de Arqueología de las Dinámicas Sociales. C/ de les Egipcíaques, 15, E-08001 Barcelona. jfgibaja@imf.csic.es

Millán Mozota

Institució Milà i Fontanals (IMF-CSIC), Grupo de Arqueología de las Dinámicas Sociales. C/ de les Egipcíaques, 15, E-08001 Barcelona. millanm@imf.csic.es

GerARD REMOLINS

ReGiraRocs, SLU, Recerca, Conservació i Difusió del Patrimoni Cultural i Natural dels Pirineus. Av. Carlemany, 115, AD700 Escaldes-Engordany, Principado de Andorra. info@regirarocs.com

ARIADNA NiETO

Institució Milà i Fontanals (IMF-CSIC), Grupo de Arqueología de las Dinámicas Sociales. C/ de les Egipcíaques, 15, E-08001 Barcelona. anietoespinet@imf.csic.es

XAVIER OMS

Universitat de Barcelona. Departament d'Història i Arqueologia. Secció de Prehistòria i Arqueologia. C/ Montalegre, 6, E-08001 Barcelona. xavieroms@gmail.com

SANTiago Higuera

Casal de Barri Folch i Torres. C/ Reina Amàlia, 31, E-08001 Barcelona. santiagohiguerag@gmail.com

VANESSA GUZMÁN

Departament d'Educació de la Generalitat de Catalunya. C/ del Marquès de Comillas, 67-69, E-08202 Sabadell.

vlopez33@xtec.cat

Miriam Cubas

Sociedad de Ciencias Aranzadi-Universidad de York. Zorroagagaina, 11, E-2014 Donostia-San Sebastián.

mcubas.morera@gmail.com

ARACELI GonZÁlez

Institución Milà i Fontanals (IMF-CSIC). C/ de les Egipcíaques, 15, E-08001 Barcelona. araceligonzalez@imf.csic.es

NicCOLÒ MAZZUCCO

Institució Milà i Fontanals (IMF-CSIC), Grupo de Arqueología de las Dinámicas Sociales. C/ de les Egipcíaques, 15,

E-08001 Barcelona. nicco.mazzucco@gmail.com

Olga PALma

ReGiraRocs SLU, Recerca, Conservació i Difusió del Patrimoni Cultura i Natura dels Pirineus. Av. Carlemany, 115, AD700

Escaldes-Engordany, Principado de Andorra. olgapalmadelcerro@gmail.com

Silvia VALENZUELA

Institució Milà i Fontanals (IMF-CSIC), Grupo de Arqueología de las Dinámicas Sociales. C/ de les Egipcíaques,

15, E-08001 Barcelona. svalenzuela@imf.csic.es 
Los programas de divulgación científica parecen preparados para todo tipo de público. Sin embargo, ciertos colectivos - p.e., personas con discapacidad física e intelectual, de la tercera edad, en riesgo de exclusión social, inmigrantes recién llegados-quedan habitualmente al margen de tales programas y no se piensa en ellos a la hora de diseñarlos. En este artículo presentamos dos actividades divulgativas centradas en la arqueología prehistórica y específicamente diseñadas para personas con discapacidad intelectual. Creemos que la metodología empleada, la evaluación realizada y los resultados obtenidos pueden servir a otros grupos de investigación o a educadores que trabajan con estos colectivos.

\section{PALABRAS CLAVE}

DIVULGACIÓN, ARQUEOLOGÍA, PERSONAS CON DISCAPACIDAD

Scientific dissemination programs seem prepared for all types of audiences, but certain groups are often left out because specialists do not think about them when planning them. Those groups include people with physical and intellectual disabilities, older adults, people at risk of social exclusion and newcomers. In this article, we present two outreach activities on Prehistory specifically designed for people with intellectual disabilities. We believe that the model used, the evaluation carried out and the results obtained can be useful for other research groups or educators who work with people with intellectual disabilities.

\section{KEYWORDS}

SCIENTIFIC DISSEMINATION, ARCHEOLOGY, PEOPLE WITH DISABILITIES

\section{Introducción y objetivos}

Se llama divulgación científica al conjunto de actividades que interpretan y hacen accesible la investigación y el conocimiento científico al público en general, a través de discursos fácilmente comprensibles para los destinatarios (Sánchez y Roque, 2011).

En el caso de la arqueología, la divulgación y la transferencia de conocimiento en el marco de un paraguas social amplio cada día tiene mayor relevancia y adeptos. Ello se refleja en el número de investigadores/as que dedican parte de su tiempo a la difusión, la proliferación de asignaturas y profesores/as universitarios/as que imparten clases sobre esta temática o los cada vez más habituales congresos y seminarios monográficos (DíazAndreu et al., 2016; Comendador, 2018). La importancia de la divulgación ha hecho que muchas publicaciones dedicadas a estas cuestiones se hayan llenado de calificativos como "arqueología pública», "arqueología comunitaria», "democratización de la comunicación arqueológica», etc. (Schadla-Hall, 1999; Ascherson, 2000; Matsuda y Okamura, 2011; Almansa, 2013; Almansa et al., 2015; Richardson y Almansa, 2015; Moshenka, 2017).

En el año 2013 un pequeño grupo de investigadores/as del CSIC, educadores/as y agentes sociales decidimos iniciar un camino muy particular en este campo de la difusión. Consistía en llevar la arqueología, la prehistoria y el conocimiento/conservación del Patrimonio a barrios de Barcelona estigmatizados debido a problemas sociales y a una población muy diversa en cuanto a su origen, costumbres y religiones (Gibaja et al., 2016a, 
2016 y y 2017). En aquel entonces, cuando configurábamos nuestras primeras actividades, la idea era construir una nueva forma de divulgar, con un relato diferente al que se explicaba en los museos. Entendíamos que tales museos no solo tenían un amplio programa de actividades vinculadas a sus propuestas expositivas, sino que, además, su público solía estar constituido por escolares, familias de clase media-alta con un nivel educativo importante y turistas. Por lo tanto, nuestro objetivo era el de conseguir estructurar algo distinto, sin cerrarnos a ciertos sectores de la población.

Cuando organizamos las primeras actividades, teníamos el convencimiento de estar llegando a todo tipo de público. Sin embargo, al poco tiempo fuimos conscientes de las carencias, debido a la exclusión de ciertos colectivos; como, por ejemplo, el de las personas con discapacidad, ciertos sectores de la tercera edad y personas en riesgo de exclusión social. Asumiendo el error inicial, acudimos a los programas divulgativos de los centros especializados — principalmente museos y parques arqueológicos- para consultar las metodologías, los modelos y las herramientas que ellos utilizaban. Para nuestra sorpresa, pudimos constatar que había un olvido patente y generalizado de tales colectivos: no había acciones específicas dedicadas a ellos, el lenguaje de las exposiciones no estaba adaptado y no se disponía de personal cualificado.

Esto nos obligó a realizar un importante trabajo de autocrítica al darnos cuenta de que los objetivos de nuestro proyecto no se podían llevar a cabo en la situación en la que estábamos. Por eso, en el año 2017 decidimos solicitar a la Fundación para la Ciencia y la Tecnología (FECYT), dependiente del Ministerio de Ciencia, Innovación y Universidades del Gobierno de España, un proyecto dedicado exclusivamente a tres de esos colectivos «olvidados» por la divulgación científica: las personas con discapacidad, las de la tercera edad y los inmigrantes recién llegados a nuestro país.

El presente trabajo se centra en el modelo de divulgación que hemos realizado con personas con discapacidad intelectual (síndrome de Down, trastornos del espectro autista, etc.). A lo largo de este artículo explicaremos la metodología empleada, las actividades llevadas a cabo y los resultados obtenidos. Este colectivo de personas con discapacidad intelectual está tan al margen de la divulgación que ni siquiera ellas mismas o sus familiares piensan que la ciencia sea un ámbito al que puedan tener acceso.

Esta concepción nos parece especialmente grave, ya que en la Convention on the Rights of Persons with Disabilities (2006), y en su equivalente en España conocido como Código de la Discapacidad (2017), se dice en el preámbulo (pág. 3): «Recognizing the importance of accessibility to the physical, social, economic and cultural environment, to health and education and to information and communication, in enabling persons with disabilities to fully enjoy all human rights and fundamental freedoms».

Aunque las instituciones políticas, sociales y culturales deberían trabajar para garantizar el cumplimiento de este código, la realidad con la que nos encontramos es otra. La exclusión de estos colectivos se ha normalizado tanto que son sistemáticamente obviados cuando se diseñan y confeccionan los programas divulgativos. Sorprende este "olvido» hacia las personas con discapacidad teniendo en cuenta los datos publicados por la 
Comisión Europea en 2010. En ellos se advertía que a una de cada seis personas de la UE se le ha diagnosticado algún tipo de discapacidad leve o grave, lo que supone unos 80 millones de ciudadanos/as (Romero, 2018).

Con el objetivo de ir supliendo estas deficiencias, y entendiendo que cada uno de nosotros debe sentirse responsable a la hora de aportar su granito de arena, en el marco del proyecto Integra>Ciencia: Divulgación científica sin barreras (FCT-17-11972), hemos organizado un conjunto de actividades didáctico-divulgativas alrededor de la arqueología.

Desafortunadamente conocíamos pocas experiencias similares (Phillips y Gilchrist, 2012; Kajda et al., 2015), por lo que al inicio nos fue difícil saber cuál era la mejor manera de organizar las actividades y de evaluar las respuestas que ofrecían los y las participantes en función de diversos condicionantes, como su discapacidad, su edad o su recorrido educativo y estimulativo. Fueron muy positivas, en cambio, las propuestas que leímos de compañeros/as de otras disciplinas que llevaban varios años trabajando con estos colectivos. Sus experiencias han sido un referente para nosotros (Gómez et al., 2014; Iglesias et al., 2015 y 2017; Fesharaki et al., 2016; Herrero et al., 2017). A este respecto, quisiéramos resaltar también el trabajo que realizan los amigos de PDICiencia (https://www.facebook. com/PDIciencia) y con los que en gran parte nos identificamos.

\section{Método y evaluación de los resultados}

\subsection{Público}

Las personas con discapacidad intelectual con las que hemos trabajado están vinculadas a la Asociación Down Catalunya (http://sindromedown.cat/es), al Grupo Cooperativo TEB (http://www.teb.org/es) y al Taller Sant Jordi de Ciutat Vella de Barcelona. Esperamos que los ejemplos que exponemos a continuación puedan servir de modelo a otros investigadores/as o educadores/as que quieran llevar la ciencia a colectivos similares.

En este sentido, hemos trabajado con personas que tienen distintas discapacidades, lo que conlleva que su grado de comprensión y atención haya sido muy variable y diverso. Esa circunstancia, que a priori podía ser un problema, se diluyó gracias al apoyo de los educadores/as y a la ayuda que se ofrecían entre sí. Siendo conscientes de las mayores dificultades que presentan algunas de esas personas, decidimos trabajar a menudo de manera individualizada, prestando atención a sus expresiones y su lenguaje corporal para saber reaccionar cuando apreciábamos que se habían desconectado del discurso que estábamos desarrollando.

Otro factor es que nuestro público presentaba edades muy diversas, desde chicos/as muy jóvenes, de 18-20 años, hasta personas mayores de 60. Esto implicaba varios hándicaps: un nivel educativo y unos intereses muy diferentes, unos conocimientos sobre determinados aspectos que dependían de su experiencia personal (por ejemplo, nos hemos 
encontrado con personas que han trabajado en la agricultura y que reconocían los productos que les mostrábamos cuando les hablábamos del neolítico) o un nivel de atención e implicación diversa que podían estar relacionados con el grado de estimulación cognitiva que han recibido a lo largo de su vida.

En el curso de las distintas actividades, hemos aprendido que la divulgación científica no puede llevarse a cabo sin la colaboración de las familias y las asociaciones relacionadas con estos colectivos. Son ellas las que mejor conocen sus necesidades, comportamiento, intereses, potencialidades, limitaciones, anhelos... y la mejor manera de transmitirles nuestros conocimientos.

En general, todas las actividades que hemos realizado han pasado por tres fases:

Fase 1. Confianza y participación. En la presentación de las actividades, establecíamos un ambiente positivo y de confianza con el público, educadores/as e investigadores/as. Ello facilitaba la participación y complicidad de todos los actores/as.

Fase 2. Promoción de los valores a través de las actividades. Se incentivaba su autoestima, valoración colectiva y la adquisición de nuevos conocimientos, así como la integración y la cooperación entre participantes.

Fase 3. Transferencia de conocimientos. Pretendíamos que ellos/as acabaran transmitiendo los conceptos, experiencias y conocimientos adquiridos a su círculo social más cercano.

\subsection{Lenguaje y herramientas didácticas}

Partimos del principio de que el lenguaje empleado debe ser siempre muy sencillo y claro. Aunque este es uno de los aspectos básicos en cualquier actividad divulgativa, la situación se complica con muchas personas con diversidad cognitiva, ya que les es difícil comprender y retener ciertos conceptos abstractos o complejos, como es el caso del tiempo histórico. Pero más allá del rigor en los contenidos científicos, también debemos emplear un lenguaje muy cuidadoso con ciertos temas, como son las relaciones y los roles de género, las creencias religiosas, las diferencias culturales y sociales, etc.

Esa dificultad a la hora de asumir ciertos conceptos se mitiga a través del tipo de actividades participativas que organizamos y de las herramientas sensitivas que empleamos. En este sentido, la primera de las actividades, como vamos a ver a continuación, tenía por objetivo hacerles entender qué era un yacimiento arqueológico, cómo funciona, cómo trabajan los arqueólogos/as y qué se encuentra. Por ello, lo más fácil fue llevarlos/as a trabajar con nosotros a una excavación real, o acompañarlos/as a un museo, como es el de las Minas Prehistóricas de Gavà, donde existe una excavación vinculada al propio recorrido del museo.

La segunda de las actividades versaba sobre los instrumentos de la prehistoria y los productos que se consumían. Para que asumieran los conceptos que queríamos transmitirles utilizamos unas maletas didácticas con réplicas de objetos (fig. 1), así como algunos de los alimentos que se consumían en la prehistoria o los restos óseos de los animales que 


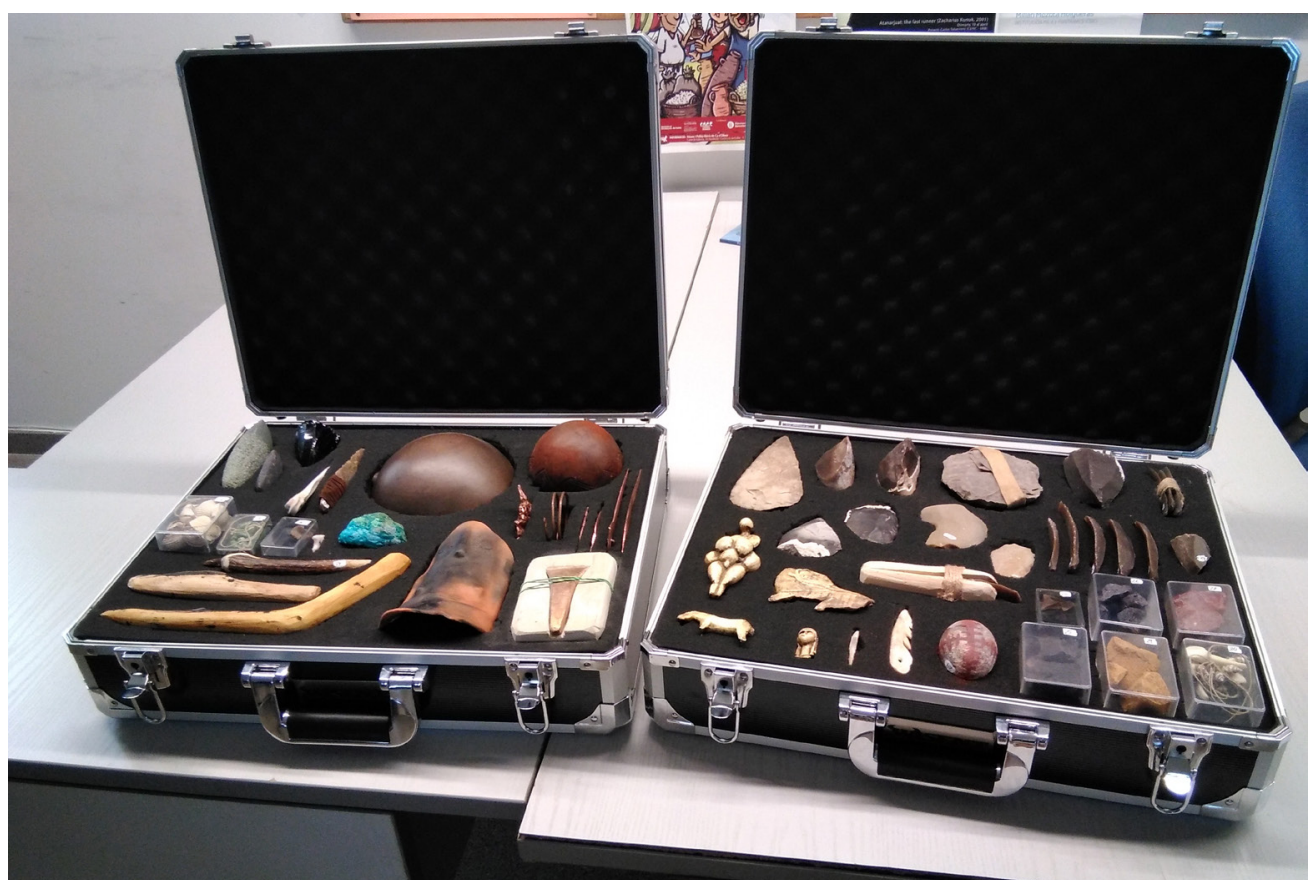

Figura 1. Maletas didácticas elaboradas por Arqueolític. Se muestran réplicas relacionadas con el Paleolítico, el Neolítico y la Edad de los Metales.

domesticaban o cazaban (fig. 2). A partir de estos datos, les propusimos hacer un trabajo culinario: una serie de platos con esos mismos productos de la prehistoria.

De esta manera, lo que les explicábamos no solo era asumido y comprendido de manera más rápida, sino que además permanecía en el tiempo; lo detectamos en la actividad de la excavación arqueológica, en tanto que a varios/as de los/as participantes que ya habían venido en la primera ocasión no fue necesario repetirles ciertas informaciones.

\subsection{Evaluación}

Los mecanismos de evaluación que hemos utilizado tienen carácter cualitativo y cuantitativo. En relación con la valoración cualitativa, cabe decir que todas las actividades han sido fotografiadas o grabadas en vídeo para poder llevar a cabo un análisis crítico. Por otra parte, hemos contado con la auditoria de una psicopedagoga que estuvo en buena parte de las actividades evaluando nuestro modo de explicar los contenidos, las reacciones del público y sus respuestas. Todo ello nos ha facilitado tener una mejor comprensión de las incidencias surgidas durante la actividad, y potenciar aquellos aspectos que mejores resultados daban, desechando o modificando los no satisfactorios. 


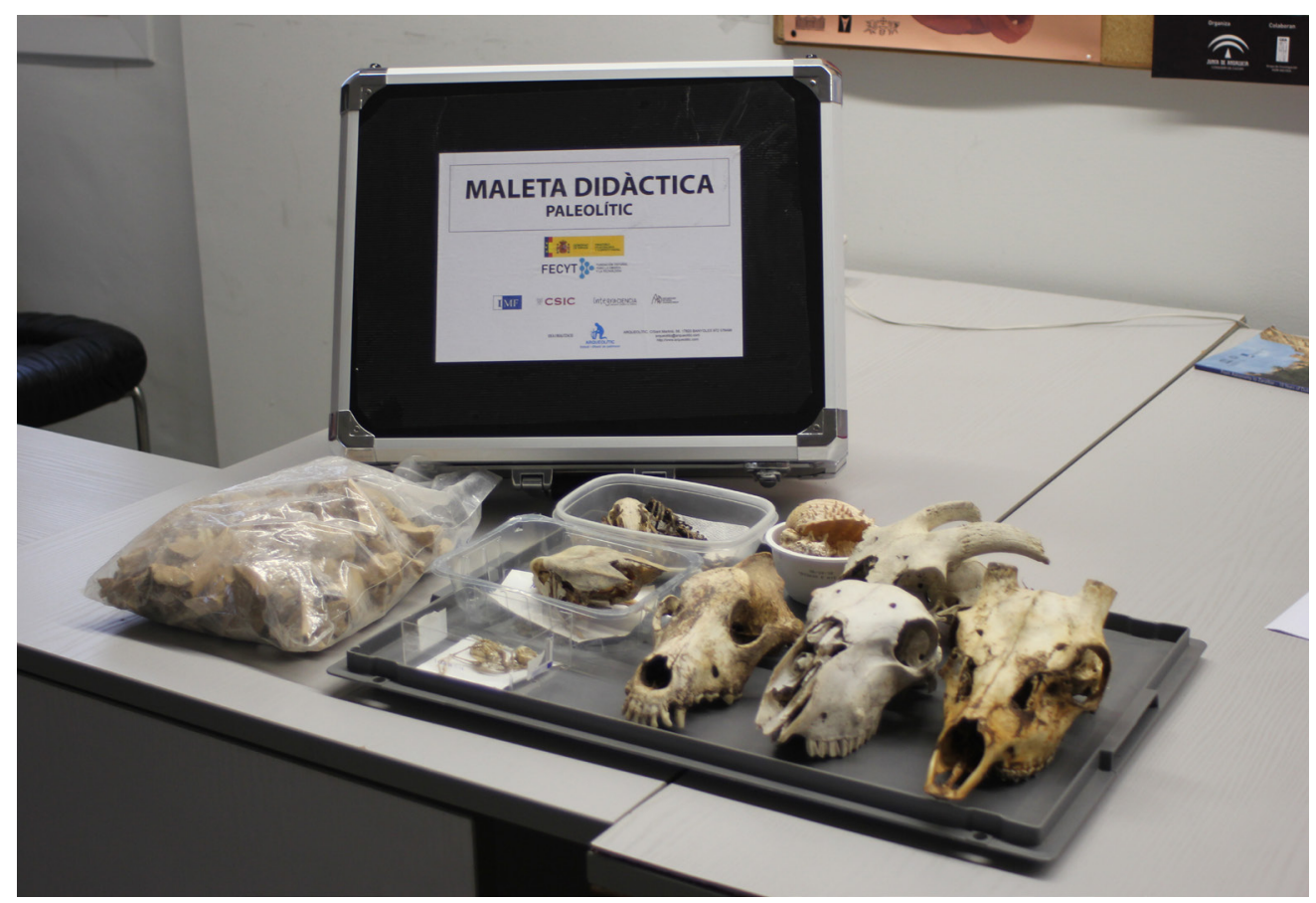

Figura 2. Restos óseos de animales empleados durante las actividades.

En lo cuantitativo, hemos elaborado una encuesta muy sencilla que debían rellenar junto a sus educadores/as en su propio centro. De esta manera evitábamos que se sintieran mediatizados/as si la cumplimentaban ante nosotros. En esa encuesta se hace una serie de preguntas sencillas que pueden responderse mediante comentarios o marcando unas casillas en las que aparecen una cara alegre y otra triste, ya que algunas de estas personas tienen muchas dificultades a la hora de expresarse por escrito.

Finamente, hicimos un seguimiento del número de visitas a nuestra web (https:// cienciainclusiva.wordpress.com/) y de las noticias que salieron en los medios de comunicación. El objetivo era analizar el grado de repercusión mediática que habían tenido nuestras actividades.

\section{Casos de estudio: propuestas de actividades}

Como hemos dicho al principio, el conjunto de actividades que organizamos se aleja de los programas divulgativos que suelen ofrecer los museos o los centros educativos. Por un lado, nuestro contexto habitual de actuación son los centros socioeducativos a los que estas 
personas acuden habitualmente, así como los centros de investigación y las excavaciones arqueológicas donde trabajan los científicos.

Por otro lado, es importante recalcar que siempre se intenta que los protagonistas de la actividad no sean los investigadores e investigadoras, sino las personas que participan; de esta manera conseguimos un contexto conocido, cercano y de confianza, en el que los y las participantes son quienes desarrollan la actividad y, en muchos casos, quienes transmiten a familiares y amigos aquello que han aprendido.

A modo de ejemplo, vamos a explicar dos modelos de actividades que hemos realizado. En ambos casos, se trata de acciones que necesitan de una preparación previa y un trabajo colaborativo con familiares y educadores. Así, explicaremos nuestra experiencia en relación con:

1) La participación en las tareas que se realizan en un yacimiento arqueológico real.

2) La elaboración de un programa de cocina prehistórica con visita final a un museo.

En ambos casos el objetivo perseguido es que, a través de estas acciones sensitivas y participativas, el público adquiera un conjunto de conocimientos que pueda interiorizar y recordar en el futuro. No queríamos que fuera simplemente una actividad lúdica, sin contenido científico, en la que lo aprendido se diluya al poco tiempo de acabar la actividad.

\subsection{Visita y participación en un yacimiento arqueológico}

Una manera dinámica, activa y sencilla de poder explicar el trabajo arqueológico (cómo se realiza, para qué sirve o sus objetivos) es invitar al público a una participación directa en una excavación arqueológica. Es habitual que en los museos se preparen cajas o recintos llenos de arena en cuyo interior se depositan unas réplicas arqueológicas que se deben buscar. Aunque se intenta simular una excavación, creemos que los resultados suelen ser pobres, porque los/as participantes acaban sacando lo más rápidamente posible los objetos como si de un concurso se tratara, olvidando cualquier tipo de rigor científico y sin seguir una metodología arqueológica real. Creemos que esta es una de las típicas actividades en las que se acaba perdiendo el objetivo de la acción, pues el contenido científico se ha difuminado en una experiencia puramente lúdica de ir a por el objeto o "en busca del tesoro».

Gracias a la colaboración del Dr. Xavier Oms, de la Universitat de Barcelona, así como de la de su equipo, organizamos dos jornadas en años consecutivos - 2017 y 2018 - en las que un grupo de personas con síndrome de Down participó en una excavación arqueológica real. En este caso, la actividad se desarrolló en el yacimiento neolítico de Les Guixeres de Vilobí (Sant Martí Sarroca, Barcelona).

La actividad se inició algunos días antes, pues se les envió un dossier donde les explicábamos el tipo de yacimiento en el que iban a trabajar, su cronología, los objetivos de la excavación, la metodología empleada y los posibles materiales que encontrarían. También 


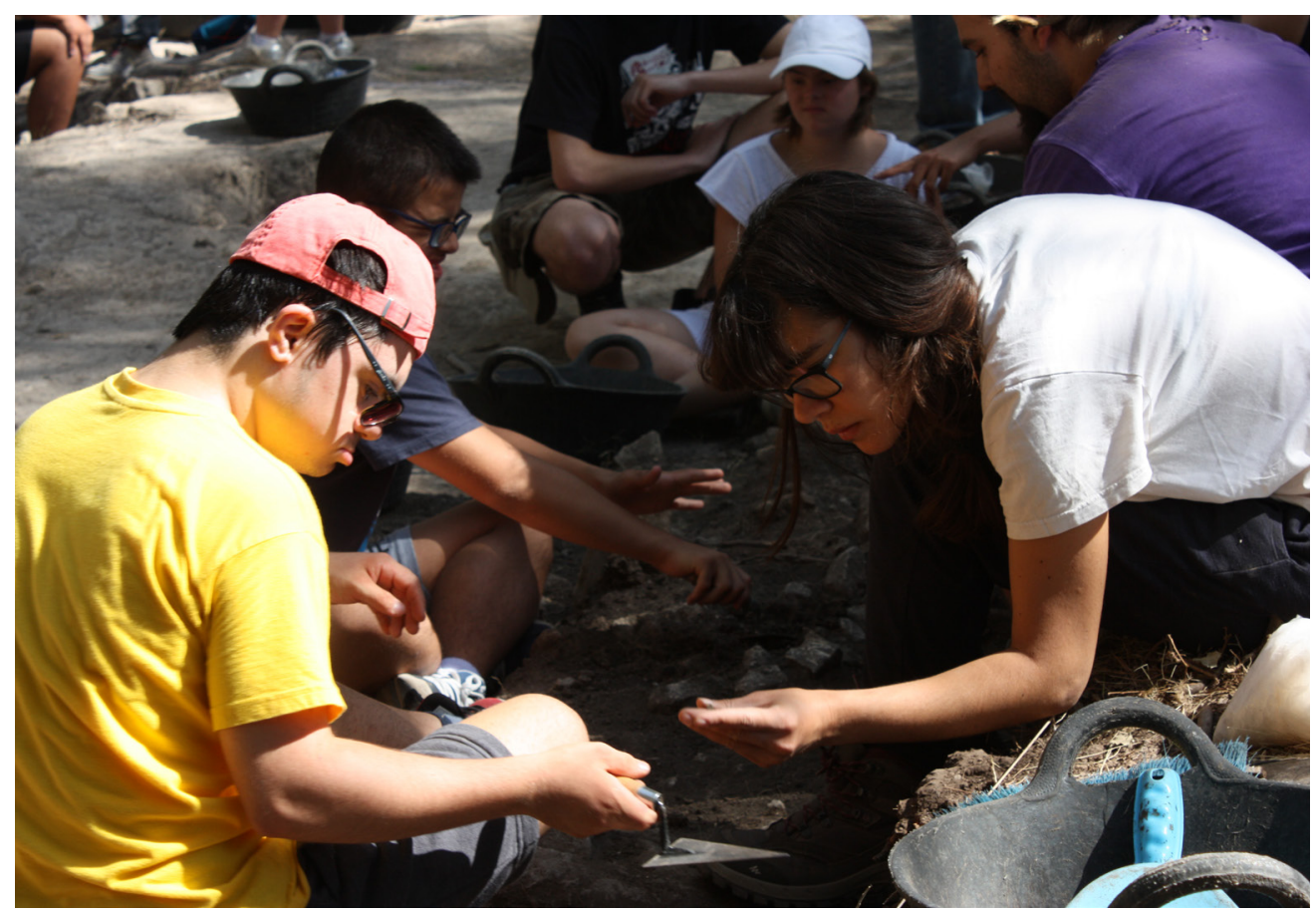

Figura 3. Momento en el que un joven con síndrome de Down y una arqueóloga están excavando en el yacimiento neolítico de Guixeres de Vilobí.

los invitábamos a visitar varias páginas web donde se hablaba del yacimiento, y les proponíamos que junto a sus familias hicieran una búsqueda de información a modo de trabajo de investigación; de esta manera llegaban al yacimiento con unos conocimientos previos.

El día de la excavación arqueológica, cada participante tenía asignado un/a arqueólogo/a. De esta manera conseguíamos que hubiera colaboración y aprendizaje individualizado. Se les explicó múltiples aspectos de la excavación, incluyendo las características del yacimiento, las herramientas necesarias para excavar y su uso, el método de excavación, las razones de cuadricularlo, la necesidad de cribar el sedimento, la forma de localizar los objetos en el espacio, etc. Al cabo de poco tiempo observamos que en la mayoría de los casos se había generado una relación y una complicidad excelente entre ambos grupos (figs. 3 y 4 ).

Por su parte, los familiares y amigos estaban fuera del área de excavación, viendo el trabajo realizado por sus hijos e hijas, preguntando al equipo de arqueológos/as infinidad de cuestiones sobre el yacimiento mismo, sobre la prehistoria o sobre las maneras de excavar. Por consiguiente, aunque pudiera parecer que los familiares tenían un papel de simples acompañantes, la realidad fue muy distinta, ya que el equipo de arqueólogos/as y sus propios hijos/as les transmitieron una batería de conocimientos. 


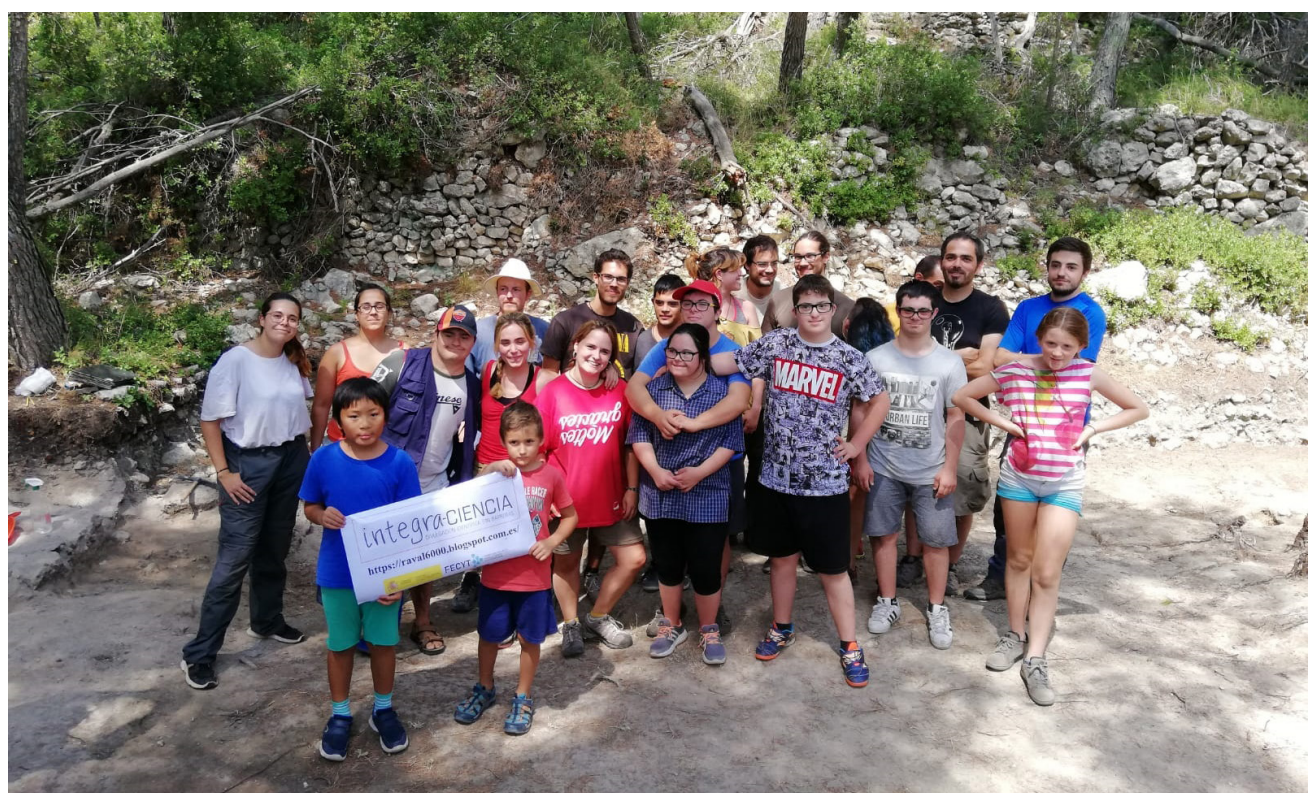

Figura 4. Personas con Síndrome de Down que, juntos a los arqueólogos, han participado en la jornada de excavación del yacimiento neolítico de las Guixeres de Vilobí.

Consideramos que esta es una manera sencilla, práctica y vivencial mediante la cual se les explica a los chicos y chicas aquello que previamente les habían mostrado sus familiares en casa, o de manera sucinta en la escuela. Pero, además, el aprendizaje acabó siendo mutuo, ya que gracias a ellos/as nosotros/as pudimos reconocer cuáles eran los aspectos que más les interesaban, que a menudo están alejados de aquello que nosotros/as consideramos relevante.

Esta primera experiencia nos ayudó sobremanera en actividades posteriores. Tras evaluarla, mejoramos los conceptos y las herramientas para acercarnos más a sus intereses, sin perder de vista el objetivo final de transmitirles ciertos conocimientos sobre la prehistoria.

\subsection{La cocina como medio de aproximación a la prehistoria}

En esta segunda actividad que presentamos hubo también una fuerte relación entre la adquisición de conocimientos y las actividades práctico-sensitivas, sin perder contenido científico. Dicha actividad se organizó con el Grupo Cooperativo TEB y el Taller Sant Jordi en torno a la cocina prehistórica, y se estructuró de la manera siguiente:

- En primer lugar, las educadoras de estas entidades, junto a las personas que participarían en nuestro taller, buscaron en Internet y en libros información sobre la pre- 


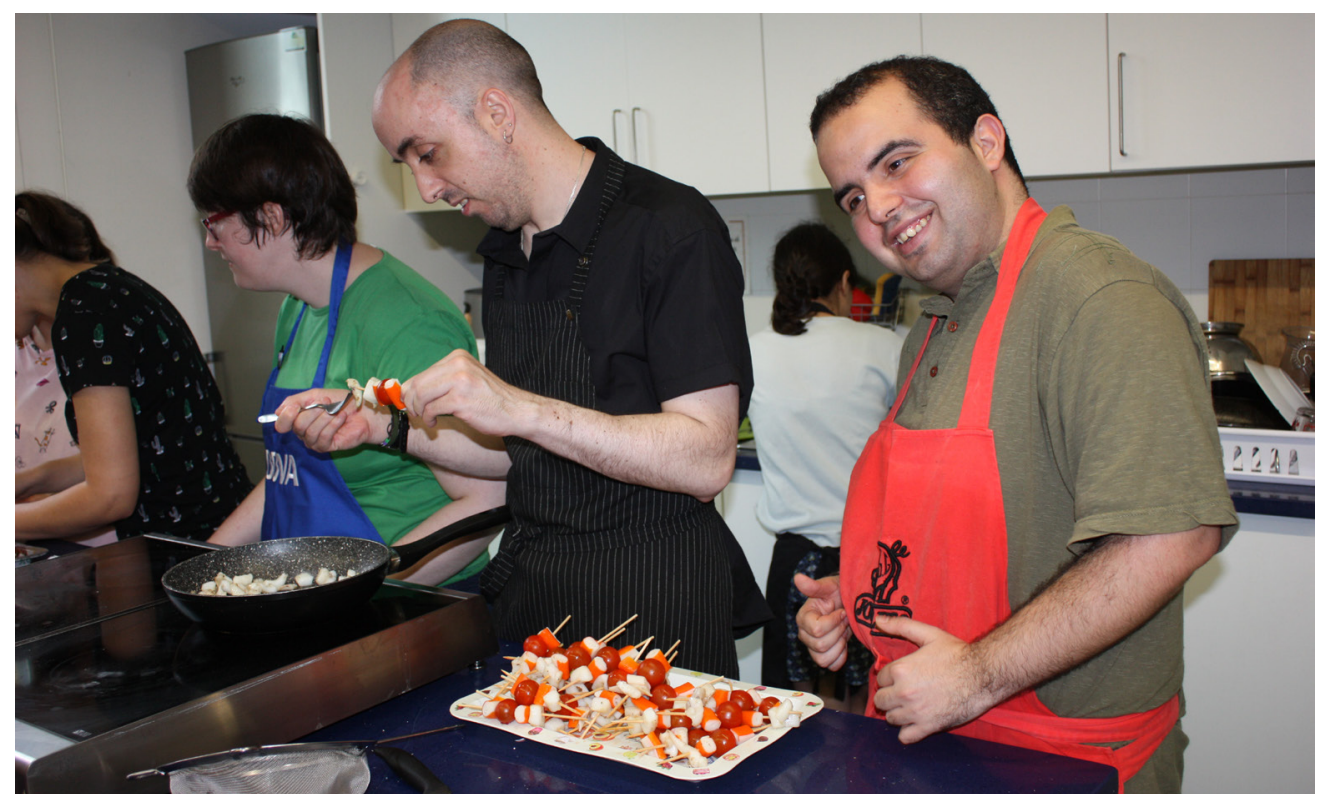

Figura 5. Personas del grupo cooperativo TEB en el taller de cocina prehistórica.

historia. Tuvieron especial interés en conocer qué posibles alimentos consumían las sociedades prehistóricas en el pasado.

- En segundo lugar, esos chicos y chicas visitaron nuestro centro de investigaciones (la Institució Milà i Fontanals del Consejo Superior de Investigaciones Científicas, IMFCSIC). Allí se les impartió una pequeña clase sobre conceptos muy genéricos de la prehistoria y se organizó un taller donde mostramos varias réplicas arqueológicas: instrumentos líticos, útiles óseos y múltiples huesos pertenecientes a distintos animales. De esta manera pudieron tocar aquello que habían visto en los libros, en Internet o a que nos habíamos referido en nuestra propia charla. La tercera de las actividades fue bastante similar al anterior, pero en este caso consistió en una conferencia sobre los productos que consumían las sociedades neolíticas. Esta charla se acompañó de productos que pudieron tocar, oler e incluso comer: cereales, ciertas legumbres, frutos silvestres, etc.

- Ya con esos primeros conocimientos y un listado de productos que les ofrecimos, el grupo y sus educadoras prepararon un conjunto de creaciones culinarias que fueron presentadas antes sus familiares, amigos/as y personal de sus entidades. Ellos/as mismos/as fueron explicando cada uno de los platos cocinados, especificando los productos que habían utilizado. En aquellos casos en los que no se usó un producto originario de la prehistoria europea se daba la explicación correspondiente: caso del tomate. Esta propuesta fue interesante porque la toma de conciencia de los errores les sirvió, precisamente, para identificar, asimilar y diferenciar mejor cuáles eran o no correctos (figs. 5 y 6). 


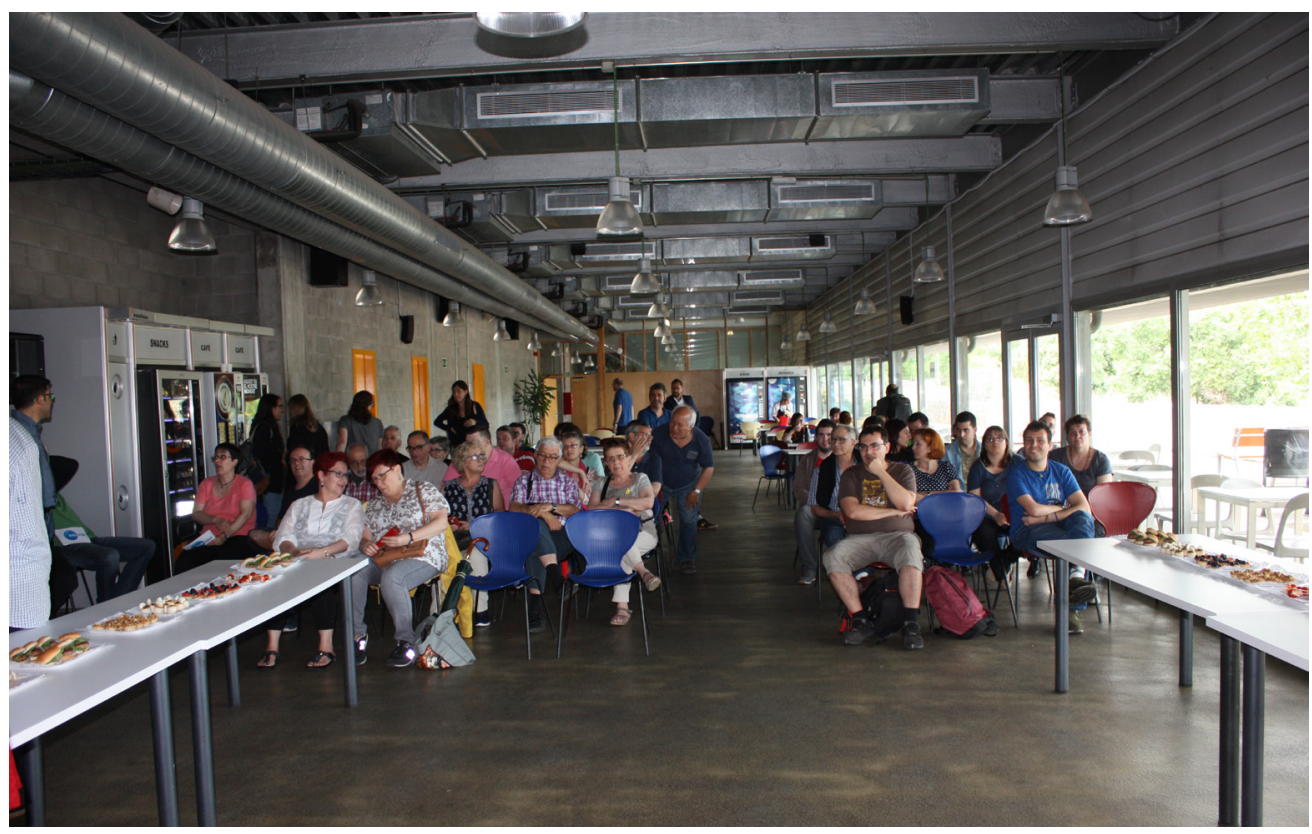

Figura 6. Muestra gastronómica de las personas que han participado en el taller de cocina prehistórica frente a familiares y trabajadores de la cooperativa TEB.

- La última actividad fue llevarlos/as a un contexto arqueológico real, cosa que solo fue posible con el grupo de la cooperativa TEB. En este caso al Parque Arqueológico de las Minas de Gavà, en la provincia de Barcelona, donde hay una excavación en funcionamiento. Esto les permitió observar, no solo cómo se estructura y organiza un museo de prehistoria, sino también qué pasos sigue un objeto arqueológico desde que se descubre hasta que se deposita y expone en un museo.

\section{Evaluación de los resultados}

Con estas actividades hemos conseguido que personas con discapacidad intelectual hayan adquirido ciertos conocimientos, y que ellos/as, sus familiares y educadores se sientan orgullosos/as de su trabajo. Lo vemos reflejado en la valoración realizada por la psicopedagoga que evalúa nuestras actividades y en las respuestas que los participantes dieron en las encuestas que les hicimos. Esta evaluación hace referencia a las actividades realizadas con las personas de la Cooperativa TEB y el Taller Sant Jordi, ya que en las excavaciones realizadas con el colectivo de la Asociación Down Catalunya aún no habíamos empezado a colaborar con la psicopedagoga. 
Tabla 1. Resultados de las encuestas realizadas a las personas de la Cooperativa TEB.

\begin{tabular}{|c|c|c|c|c|c|}
\hline & & & & No contesta & Comentarios \\
\hline $\begin{array}{l}\text { ¿Las explicaciones } \\
\text { son claras? }\end{array}$ & 14 & 0 & 0 & 0 & $\begin{array}{l}\text { Valoración positiva del soporte } \\
\text { visual (imágenes) para las } \\
\text { explicaciones. Facilitan su } \\
\text { comprensión. }\end{array}$ \\
\hline ¿Te ha gustado? & 12 & 1 & 0 & 1 & $\begin{array}{l}\text { Valoran las explicaciones claras } \\
\text { e interesantes por parte de los } \\
\text { arqueólogos. }\end{array}$ \\
\hline ¿Lo recomendarías? & 8 & 1 & 4 & 1 & $\begin{array}{l}\text { Consideran que podría ser } \\
\text { explicado a otras personas } \\
\text { de colectivos distintos. } \\
\text { Aquí hay casos que no lo } \\
\text { recomendarían, pero no } \\
\text { especifican los motivos. }\end{array}$ \\
\hline $\begin{array}{l}\text { ¿Te ha parecido } \\
\text { interesante y útil? }\end{array}$ & 11 & 1 & 1 & 1 & $\begin{array}{l}\text { Lo consideran muy interesante } \\
\text { y se muestran satisfechos } \\
\text { gracias a la utilidad que le } \\
\text { encuentran, en especial en } \\
\text { relación con el taller de cocina. }\end{array}$ \\
\hline
\end{tabular}

En las respuestas de las 14 personas encuestadas de la Cooperativa TEB, por lo general se aprecia una opinión muy positiva de las actividades. No obstante, debemos seguir trabajando y perfeccionado el discurso y las herramientas, ya que algunos encuestados/as indican que las explicaciones les han parecido claras, pero no siempre las consideran interesantes. Este hecho les ha llevado a no recomendar la actividad (tabla 1). Curiosamente, esa evaluación entra en contradicción con algunos de sus comentarios, puesto que expresan que están deseando seguir trabajando y descubriendo otros aspectos de la prehistoria.

Las personas encuestadas del Taller Sant Jordi fueron 15. A diferencia del caso anterior, las opiniones fueron mucho más positivas (tabla 2). Prácticamente todos/as han entendido las explicaciones, les han gustado las actividades, las recomendarían y les han parecido útiles. Aquí, más del 95\% responde de manera concreta y sin añadir ningún comentario, probablemente porque a muchos/as de ellos/as les cuesta expresarse por escrito.

Estos datos nos demuestran que, a pesar de su diversidad funcional, edades diferentes o nivel educativo alcanzado, hemos conseguido atraer su atención y alcanzar nuestros objetivos.

Con este trabajo divulgativo, hemos demostrado que es posible y necesario trabajar con estos colectivos. Hay que tener claro que la «no-capacidad» no proviene de sus barre- 
Tabla 2. Resultados de las encuestas realizadas a las personas del Taller Sant Jordi.

\begin{tabular}{|c|c|c|c|c|}
\hline & & & & Comentarios \\
\hline $\begin{array}{l}\text { ¿Las explicaciones } \\
\text { son claras? }\end{array}$ & 15 & 0 & 0 & $\begin{array}{l}\text { Necesidad de explicaciones más lentas } \\
\text { y extensas }\end{array}$ \\
\hline ¿Te ha gustado? & 14 & 1 & 0 & Sin comentarios \\
\hline ¿Lo recomendarías? & 14 & 1 & 0 & Sin comentarios \\
\hline $\begin{array}{l}\text { ¿Te ha parecido } \\
\text { interesante y útil? }\end{array}$ & 13 & 2 & 0 & Sin comentarios \\
\hline
\end{tabular}

ras, sino de nuestra incapacidad de ofrecer los medios y los recursos idóneos para llegar a sus potencialidades. La manera de explicar los conceptos y las herramientas didácticas adaptadas les permiten adquirir, entender y disfrutar de la ciencia.

Por otra parte, aunque es difícil cuantificar y evaluar la repercusión que han tenido nuestras actividades en los medios de comunicación y las redes sociales, algunos datos nos indican la buena acogida que ha tenido nuestro proyecto. A este respecto, la noticia de la excavación arqueológica con personas con síndrome de Down fue publicada en El Periódico por Michele Catanzaro, uno de los periodistas más conocidos en el ámbito de la divulgación científica. En relación con la actividad de la cocina, fue grabada y emitida por la televisión digital TEBvist, medio dedicado a las personas con discapacidad intelectual (https://youtu.be/CjFo3hkD-8U). Finalmente, en la web de nuestro proyecto algunas de las entradas más visitadas son las relacionadas, precisamente, con las actividades efectuadas con estos colectivos (tabla 3).

Tabla 3. Número de visitas recibidas en nuestra web relacionadas con las actividades efectuadas con los colectivos con los que hemos trabajado: Asociación Síndrome de Down Catalunya, Cooperativa TEB y Taller Sant Jordi (https://cienciainclusiva. wordpress.com/).

\begin{tabular}{lr}
\hline & Visitas \\
\hline Reportaje Youtube: excavación Asoc. Down Catalunya & 620 \\
Actividad: excavación arqueológica 2017 Asoc. Down Catalunya & 380 \\
Actividad: excavación arqueológica 2018 Asoc. Down Catalunya & 802 \\
Actividad: visita yacimiento arqueológico Cooperativa TEB & 64 \\
Actividad: conferencia Cooperativa TEB & 295 \\
Actividad: Taller Sant Jordi & 169 \\
Total & 2330 \\
\hline
\end{tabular}




\section{Conclusiones}

Qué mejor manera de trasladar la ciencia al conjunto de la ciudadanía que llevándola a su propia casa, a los centros socioeducativos que visitan diariamente y a los lugares donde trabajan los investigadores/as. Trabajar en su propio ámbito facilita el aprendizaje y la comunicación.

Como dijimos al principio, entendemos que la ciencia debe llegar a todos los sectores de la sociedad, independientemente de la edad de las personas, sexo-género, estudios o capacidades adquisitivas, intelectuales y/o físicas. Los científicos/as, educadores/as, psicopedagogos/as, políticos/as... debemos conseguir que todas las personas puedan disfrutar de la ciencia (Verdugo et al., 2018). Pensamos que son este tipo de actividades de carácter más práctico, sensitivo y participativo las que ofrecen los mejores resultados, si tenemos en cuenta sus opiniones y el grado de conocimientos asimilados.

El modelo divulgativo empleado es el de la cercanía, utilizando herramientas didácticas de carácter sensitivo y en el que se usa un lenguaje sencillo, respetuoso e inclusivo. Se ha priorizado un trato de confianza y de complicidad, nunca de superioridad, para evitar generar un ambiente de frustración o desmotivación. Pensar que no lo van a entender es una concepción absolutamente falsa.

El acceso de estos colectivos a los centros de investigación, y a las actividades científicas que allí se llevan a cabo, también ha sido uno de los puntos fuertes, ya que esto les permite poder familiarizarse y acercarse a un mundo que antes les parecía inaccesible y lejano. Finalmente, este modelo divulgativo ha apostado siempre por una participación activa y real, donde las personas con las que hemos trabajado se han convertido en protagonistas del proyecto.

Esto puede parecer sencillo, pero no lo es. La ayuda de los familiares, así como de los educadores/as de las instituciones que acogen a estas personas, nos ha orientado y ha corregido nuestros continuos errores. Gracias a ellos y ellas hemos conseguido que poco a poco los resultados sean mejores.

Pero, sobre todo, creemos que hemos conseguido que estos colectivos pudieran disfrutar de la ciencia como lo hacen cada día miles de personas sin discapacidad, que acuden a la escuela o a los museos. Citando las palabras de uno de los padres de un hijo con síndrome de Down: «Se nos suele invitar a fiestas o a eventos deportivos, pero nunca a actividades culturales».

No obstante, más allá de cómo hacemos divulgación científica, hay un aspecto relevante que no debemos olvidar: si las personas no entienden cómo la ciencia repercute en su vida diaria, difícilmente habrá una presión sobre los gobiernos para que aumente la inversión en investigación. La comunicación científica tiene, por tanto, un carácter pedagógico, a la vez que reivindicativo. El futuro de la ciencia depende de nuestros gobiernos, pero como científicos/as también tenemos una responsabilidad y una deuda con la sociedad. Toda investigación debiera tener implícito un trabajo divulgativo y de retorno social. 


\section{Agradecimientos}

Queremos dedicar este trabajo a la memoria de Maria Casas, vecina del barrio de El Raval de Barcelona, siempre implicada en el desarrollo y la organización del proyecto. Sin ella este proyecto no hubiera sido una realidad.

El proyecto ha sido posible gracias a la financiación ofrecida por la Fundación Española para la Ciencia y la Tecnología (FECYT): Integra>Ciencia: Divulgación científica sin barreras (FCT-17-11972).

Queremos dar las gracias especialmente a los chicos y chicas que han participado, a sus familiares, amigos/as y educadores/as de las Asociación Down Catalunya, Grupo Cooperativo TEB y Taller Sant Jordi. También expresar nuestro mayor agradecimiento a todo el equipo de arqueólogos/as de la excavación de Guixeres de Vilobí, así como a la dirección y el personal del Parque Arqueológico de las Minas de Gavà y del Museu de Ca n'Oliver de Cerdanyola del Vallès.

Por último, no queremos olvidarnos de muchas de las instituciones que han apoyado este proyecto: Institució Milà i Fontanals (IMF-CSIC), Área de Prehistoria de la Universitat de Barcelona, Casal de Barri Folch i Torres, Ajuntament de Barcelona, Cooperativa Impulsem, Ajuntament de Sant Martí Sarroca, Adhoc_Cultura, Regirarocs y La Ciència al Teu Món.

\section{Bibliografía}

ALMANSA, J., 2013, Introducción. Hablando de arqueología pública, en J. ALMANSA (ed.), Arqueología pública en España, JAS Arqueología, 3- 12 .

ALMANSA, J., GALMÉS, A., ABRUNHOSA, A., GARROCHENA, E., CARDONA, G., PALOMERO, I., SEÑORAN, J.M., APARICIO, P. y VIZCAÍNO, A., 2015, Arqueólogo busca público: políticas de integración y el futuro de la arqueología, VII Jornadas de jóvenes en investigación arqueológica. Revista Arkeogazte anexo 1, 339-369.

ASCHERSON, N., 2000, Public Archaeology, Public Archaeology 1 (1), 1-4.

COMENDADOR, B., 2018, Arqueología pública en las aulas universitarias: un primer balance de su implantación, CPAG 28, 9-24.
DÍAZ-ANDREU, M., PASTOR, A. y RUIZ, A., 2016, (eds.) Arqueología y comunidad: El valor social del patrimonio arqueológico en el siglo XXI, JAS Arqueología.

FESHARAKI, O., GARCÍA-FRANK, A., IGLESIAS ÁLVAREZ, N., GÓMEZ-HERAS, M., MARTÍNPEREA, D. y RICO, R., 2016, Diseño universal y materiales multisensoriales en las actividades de divulgación de Geodivulgar con la asociación Ciencia sin Barreras, Geo-Temas 16 (1), 729-732.

GIBAJA, J. F., HIGUERA, S. y MIRÓ, L. 2016a, "Raval, 6000 años de historia»: un proyecto para llevar la arqueología y la prehistoria a la ciudadanía, Cuadiernu: Difusión, Investigación y conservación del patrimonio cultural 4, 114-119.

GIBAJA, J.F, HIGUERA, S., MARÈS, J., BORRUT, N. y PALOMO, A. 2016b, Raval 6000 
anys d'Història: un proyecto para aproximar la prehistoria a la ciudadanía, en M. DÍAZ-ANDREU, A. PASTOR y A. RUIZ (eds.), Arqueología y comunidad: El valor social del patrimonio arqueológico en el siglo XXI, JAS Arqueología, 191-207.

GIBAJA, J. F., VILA, A., HIGUERA, S., y RICHAUD, I., 2017, RavalEsCiencia: actuaciones divulgativas en las calles del centro de Barcelona, en L. PRADOS y C. LÓPEZ (eds.) Museos arqueológicos y género. Educando en Igualdad, 451-471.

GÓMEZ, M., GONZALO, L., GARCÍA-

FRANK, A., SARMIENTO, G. N., GONZÁLEZ, L., MUÑOZ, M. B., GARCÍA, R., HONTECILLAS, D., URETA, S. y CANALES, M. L., 2014, Geología para sordociegos: una experiencia multisensorial para la divulgación de la ciencia, Serie El CSIC en la escuela: investigación sobre la enseñanza de la ciencia en el aula. Consejo Superior de Investigaciones Científicas (España) 10, 45-55.

HERRERO, S., PERLES, J., LÓPEZ, A. M., JIMÉNEZ, M. C., FERNÁNDEZ, J. M., GIBAJA, M. y ALONSO, A., 2017, ConCiencia inclusiva: talleres experimentales de crecimiento de cristales como herramienta pedagógica inclusiva, Boletín de la Real Sociedad Española de Historia Natural Sección Aula, Museos y Colecciones 4, 5-13.

IGLESIAS, N., FESHARAKI, O., GARCÍA-FRANK, A., GONZÁLEZ, L., RICO, R., SALAZAR, R. W., SACRISTÁN, S., MARTÍN, D., HONTECILLAS, D., GARCÍA, R., GÓMEZ-HERAS, M., SARMIENTO, G. N., MUÑOZ, M. B., URETA, S., CANALES, M. L. y DEL MORAL, B., 2015, Dejando huella: divulgación paleontológica para personas con diversidad funcional, Current Trends in Paleontology and Evolution, XIII EJIP Conference Proceedings, 154-162.

IGLESIAS, N., GARCÍA-FRANK, A. Y FESHARAKI, O., 2017, Ideas y reflexiones para una divulgación científica efectiva, Boletín de la Real Sociedad Española de Historia Natural, Sección Aula, Museos y Colecciones 4, 29-41.
KAJDA, K., MICHALIK, T. y KOBIALKA, D., 2015, Heritage for all. A contribution to the Inclusion of People with Intellectual Disabilities in Archaeology: A Polish Perspective, Current Swedish Archaeology 23, 131-156.

MATSUDA, A. y OKAMURA, K., 2011, Introduction: New Perspectives in Global Public Archaeology, en A. MATSUDA y K. OKAMURA (eds.), New Perspectives in Global Public Archaeology, Londres, Springer, 1-18.

MoshenKA, G., 2017, Key Concepts in Public Archaeology, UCL Press.

PHILLIPS, T. y GILCHRIST, R., 2012, Inclusive, Accessible, Archaeology, en R. SKEATES, C. MCDAVID y J. CARMAN (eds.), The Oxford Handbook of Public Archaeology, Oxford, Oxford University Press, 673-693.

RICHARDSON, L. J y ALMANSA, J., 2015, Do you even know what public archaeology is? Trends, theory, practice, ethics, World Archaeology 47(2), 194-211.

ROMERO, A., 2018, La discapacidad y el nuevo pilar europeo de derechos sociales. Siglo Cero: Revista Española sobre Discapacidad Intelectual 49 (2), 105-113.

SÁNCHEZ, Y. y ROQUE, Y., 2011, La divulgación científica: una herramienta eficaz en centros de investigación, Anales de Investigación 7, 91-94.

SCHADLA-HALL, T., 1999, Public Archaeology, European Journal of Archaeology 2 (2), 147-158.

VERDUGO, M. A., AMOR, A. M., FERNÁNDEZ, M., NAVAS, P. y CALVO, M. I. 2018, La regulación de la inclusión educativa del alumnado con discapacidad intelectual, una reforma pendiente, Siglo Cero: Revista Española sobre Discapacidad Intelectual 49 (2), 27-58. 
